\title{
ESTRUCTURA Y DIVERSIDAD DE LIANAS Y HEMIEPÍFITAS DE LA SELVA BAJA DE LA PROVINCIA DE OXAPAMPA - PASCO, PERÚ
}

\section{STRUCTURE AND DIVERSITY OF THE LIANA AND HEMIEPIPHYTE COMMUNITY IN THE LOWLAND FOREST AT OXAPAMPA, PASCO - PERÙ}

\author{
Caissa Revilla Minaya ${ }^{1}$ y Abelardo Calderón Rodríguez ${ }^{2}$
}

\section{Resumen}

Se estimaron la estructura y diversidad de la comunidad de lianas y hemiepífitas de los bosques de selva baja de la Estación Biológica Paujil, en el Parque Nacional YanachagaChemillén, y de la Comunidad Nativa Yanesha Buenos Aires, situadas en la provincia de Oxapampa - departamento de Pasco, Perú. Se evaluaron las lianas y hemiepífitas con DAP $\geq 0.5$ $\mathrm{cm}$ que estuvieron enraizadas en 3 transectos de $2 \times 500 \mathrm{~m}$, establecidos al azar en cada sitio de estudio. La hemiepífita Philodendron sp.1 fue la más importante estructuralmente en la E.B. Paujil, debido a que obtuvo los mayores valores de densidad, frecuencia y longitud relativas; Philodendron ernestii Engl. fue su contraparte en la C.N. Buenos Aires, siendo la mas densa y frecuente en esta localidad. Bignoniaceae, Fabaceae y Menispermaceae fueron las familias predominantes en ambos sitios, lo cual concuerda con lo esperado para este tipo de bosque; Araceae fue igualmente una familia importante, sobretodo en la E.B. Paujil. Se obtuvieron valores elevados del índice de diversidad Shannon para los dos lugares; el índice $\alpha$ de Fisher reveló que existe una mayor diversidad en los lugares estudiados en comparación con otras localidades en diferentes lugares del Neotrópico pertenecientes al mismo tipo de bosque. Esto puede estar relacionado con una mayor precipitación anual en la zona de estudio, lo cual indica la gran influencia de los factores ambientales en la comunidad de plantas trepadoras.

Palabras Clave: Lianas, hemiepífitas, estructura, densidad, dominancia, diversidad, Parque Nacional Yanachaga-Chemillén, Comunidades Nativas Yanesha, selva baja

\begin{abstract}
The structure and diversity of the liana and hemiepiphyte community were examined in the lowland forest of the Estación Biológica Paujil at the Parque Nacional Yanachaga-Chemillén and the Comunidad Nativa Yanesha Buenos Aires in the Oxapampa province of Pasco - Perú. Lianas and hemiepiphytes with $\mathrm{DBH} \geq 0.5 \mathrm{~cm}$ were sampled in three $2 \times 500 \mathrm{~m}$ plots, placed randomly in both sites. The hemiepiphyte Philodendron sp.1 was the most structurally important climber at E.B. Paujil, due to its high density, frequency and length values. Philodendron ernestii Engl. played a similar role in Buenos Aires, having the highest density and frequency. Bignoniaceae, Fabaceae and Menispermaceae were the predominant families at both sites, which is expected for this forest type. Araceae was also an important family, above all at the E.B. Paujil. The value of the Fisher $\alpha$ Index revealed a higher diversity at both of the present study sites in comparison to that observed at other Neotropical sites of the same forest type. This result may be due to the elevated annual rainfall at the study sites, which may demonstrate the important contribution made by environmental factors on the climbing plant community.

Key Words: Lianas, hemiepiphytes, structure, density, dominance, diversity, Parque Nacional Yanachaga-Chemillén, Yanesha Native Communities, lowland forest
\end{abstract}

\section{Introducción}

Las lianas y hemiepífitas son muy importantes en la diversidad, dinámica y regeneración de bosques tropicales. Pueden llegar a representar más del $20 \%$ de las plantas de crecimiento libre menores de 2 metros de alto en el sotobosque (Gentry, 1983; Putz, 1984b) y la cantidad de árboles infestados por ellas puede variar entre el 30 a 50\% (Putz, 1983, 1984b). La abundancia $\mathrm{y}$ diversidad de plantas trepadoras se incrementa conforme disminuyen la altitud y latitud (Putz \& Chai 1987; Hegarty \& Caballé, 1991). Así, existe una diferencia considerable de diversidad entre el bosque tropical en Panamá (DeWalt et al., 2000) y los alrededores de Manaos, Brasil (Laurance et al., 2001), teniendo 7.9 y 21.3 respectivamente, de acuerdo al índice de diversidad $\alpha$ de Fisher.

Las lianas generalmente utilizan más de un soporte, esto influencia el crecimiento y mortalidad de los árboles hospederos (Clark \& Clark, 1990; Bongers et al., 2002), condicionando también el tamaño de los claros cuando alguno de sus soportes cae (Putz, 1984 a b). Por ser también una de las 
primeras formas de vida colonizadoras de claros, resultan importantes en la dinámica de los bosques, ya que la gran abundancia de lianas puede llegar a bloquear la luz necesaria para plántulas de árboles, impidiendo su regeneración (Bongers et al., 2002). Por otro lado, las plantas trepadoras son utilizadas por los animales como alimento y también para desplazarse entre las copas de los árboles (Gentry, 1991; Emmons \& Gentry, 1983).

Las investigaciones existentes aseguran que el rol de estas plantas es fundamental para establecer el estado de conservación de los bosques, determinando si éstos han sufrido una alteración (natural o antropogénica) de acuerdo a los cambios en la estructura y composición de sus comunidades (DeWalt et al., 2000; Laurance et al., 2001). Pero se afirma también, que aún faltan realizar más estudios sobre ecología de plantas trepadoras (Pérez-Salicrup \& Sork, 2001; Burnham, 2002), incluso en el Perú, donde la información publicada sobre estas plantas es prácticamente nula.

Resulta importante entonces realizar investigaciones que describan y expliquen los patrones encontrados en las comunidades de plantas trepadoras, $\mathrm{y}$, si es posible, plantear los procesos que las rigen.

En este contexto, con este estudio se pretende describir la estructura y diversidad de lianas y hemiepífitas de los bosques de selva baja pertenecientes al Parque Nacional YanachagaChemillén y a las cercanías de la Comunidad Nativa Yanesha Buenos Aires en la provincia de Oxapampa en el departamento de Pasco, Perú.

\section{Metodología}

Área de estudio

Este estudio fue realizado en dos localidades. La primera es la Estación Biológica Paujil, situada a aproximadamente $350 \mathrm{msnm}$, en el extremo oriental del Parque Nacional Yanachaga-Chemillén (10 $35^{\circ}$ $10^{\circ} 10^{\prime} \mathrm{S}$ y $\left.75^{\circ} 10^{\prime}-75^{\circ} 45^{\prime} \mathrm{O}\right)$, en el departamento de Pasco, Perú (Figura 1). Esta área natural protegida abarca una extensión de 122000 ha, y un rango altitudinal de 300 a 4000 msnm., comprendiendo las ecorregiones Puna, Selva Alta o Yungas y Selva Baja o Bosque Tropical Amazónico (Brack, 1984), en la cual está ubicada la Estación Biológica Paujil. Por lo conversado con los guardaparques de la Estación, se puede decir que esta ecorregión se encuentra relativamente conservada, ya que si bien existen eventuales cazadores furtivos por el límite del Parque, no se ha realizado actividad extractiva de mayor escala y es de difícil acceso para las poblaciones cercanas. Según la clasificación de la ONERN (1995), la Selva Baja es llamada Bosque Húmedo Tropical (bh-T), se caracteriza por tener una temperatura promedio anual de $24^{\circ} \mathrm{C}$ y una humedad relativa superior al $70 \%$, la época de lluvias es de Diciembre a Marzo y presenta una precipitación promedio anual de
$3419.5 \mathrm{~mm}$ (dato registrado en Puerto Bermúdez [ONERN, 1995]). Se encuentra en la región edáfica Ferralsólica, la cual posee suelos de tipo rojo muy pobres, menos a lo largo de los ríos, donde los suelos son aluviales. Por no existir datos climáticos específicos de los lugares de estudio, asumiremos que la información mencionada para la ecorregión de Bosque Húmedo Tropical se aplica para ambas localidades. Sin embargo, consideraremos el dato de precipitación media anual de $4000 \mathrm{~mm}$ (Phillips \& Millar, 2002), registrado por Gentry (1991) para uno de sus transectos instalado aproximadamente entre Paujil y Buenos Aires.

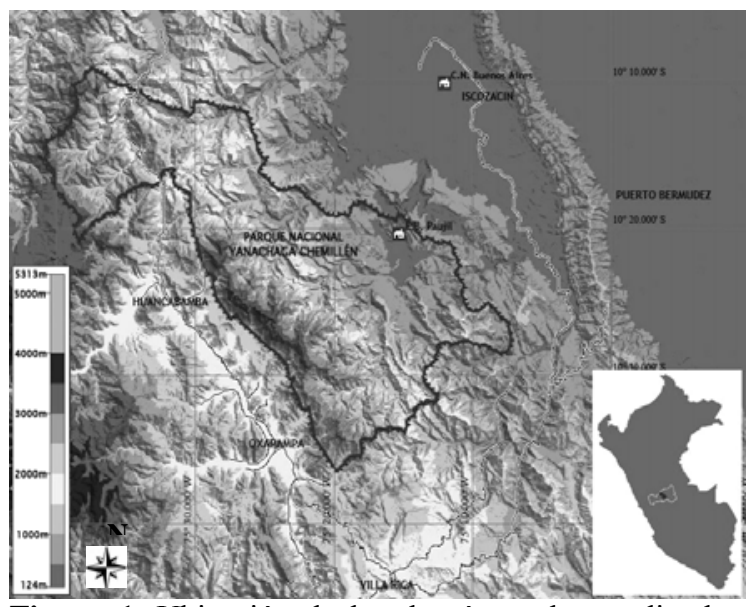

Figura 1. Ubicación de las dos áreas de estudio: la Estación Biológica Paujil, dentro del Parque Nacional Yanachaga-Chemillén, y la Comunidad Nativa Buenos Aires.

La otra parte del estudio se realizó en el bosque aledaño a la Comunidad Nativa Yanesha Buenos Aires, ubicada en el valle del río Palcazu, departamento de Pasco, Perú (Figura 1), aproximadamente a $20 \mathrm{~km}$ de la E.B. Paujil, por lo que se encuentra en el mismo tipo de bosque que la E.B. Paujil. Esta comunidad fue titulada en 1976, tiene una extensión de 3258 ha (IBC, 2006) y está conformada por aproximadamente 450 personas pertenecientes a la etnia Yanesha. El bosque que les pertenece tiene una extensión de aproximadamente 100 ha y se encuentra en la ecorregión de Selva Baja, al igual que la E.B. Paujil, a una altitud aproximada de 340 msnm. Fue declarado como reserva de la comunidad hace cerca de 20 años para protegerlo de la deforestación que en esos momentos comenzaba a ser efectuada en toda la zona.

Recolección de Datos

Se empleo como unidad muestreal el transecto Gentry (1991) de 2 X $500 \mathrm{~m}$ siendo el tamaño de muestra de 3 transectos por cada localidad instalados al azar, estando constituido cada uno de estos por 10 subunidades de 2 X 50m. El transecto Gentry es 
propuesto para abarcar un área suficientemente grande con el fin de evitar los parches de lianas de una sola especie derivados generalmente de una reproducción vegetativa (Gentry, 1991). En cada transecto se colectaron muestras botánicas de todos los individuos vivos de las especies de lianas y hemiepífitas con DAP $\geq 0.5 \mathrm{~cm}$ que estuvieran enraizados dentro del suelo. Las hemiepífitas fueron colectadas únicamente cuando sus raíces llegaban al suelo; no se consideraron aquellas que parecían haber tenido contacto con este, pero que lo habían perdido. Se registró el DAP a $130 \mathrm{~cm}$ desde el punto de enraizamiento siguiendo la contorsión del tallo de cada planta trepadora. En aquellas que se ramifican debajo de los $130 \mathrm{~cm}$ del punto de enraizamiento, se midió el DAP $20 \mathrm{~cm}$ debajo del punto de ramificación, mientras que las lianas con raíces adventicias que sobrepasaban los $80 \mathrm{~cm}$ desde el punto de enraizamiento fueron medidas $50 \mathrm{~cm}$ después de la última raíz. Para más detalle revisar los protocolos propuestos por Honorio et al. (2003) y Gerwing et al. (2006). Además del DAP se anotó también la longitud estimada.

Análisis de los datos

Con los datos obtenidos se estimaron valores absolutos y relativos de densidad, frecuencia, dominancia y longitud, para hallar índices de estructura como son el Índice de Valor de Importancia de cada especie de liana/hemiepífita (IVIL) y el Valor de Importancia de las Familias (VIFL) de las trepadoras encontradas en ambos bosques ( $\mathrm{Paz} y$ Miño, 1990 citado por Romero-Saltos, 1999). También se calcularon los índices de diversidad Shannon y $\alpha$ de Fisher; el primero suele usarse en investigaciones de ecología en general; el último ha sido ampliamente usado en estudios anteriores realizados con lianas y no está afectado por el tamaño de muestra (Berry, 2002; Magurran, 1988). Las fórmulas de los índices utilizados se encuentran en la Tabla 1.

\section{Resultados y discusión}

Estructura de las comunidades de plantas trepadoras

En la Tabla 2, se muestran los índices de valor de importancia de las plantas trepadoras de las localidades de la E.B. Paujil y la C.N. Buenos Aires respectivamente. Los resultados se analizan a continuación de acuerdo a las diferentes variables que conforman este índice.

Densidad

La E.B. Paujil presentó una densidad promedio de 142.3 ind/0.1 ha, mientras que en la C.N. Buenos Aires se encontró un promedio de 109 ind/0.1 ha. Comparamos estos resultados con los determinados por Gentry $(1985,1991)$ para sus transectos en bosques bajos tropicales. El encontró un promedio de 69 individuos. La diferencia se debe a que Gentry utilizó un diámetro mínimo de $2.5 \mathrm{~cm}$. Tomando en cuenta únicamente las plantas trepadoras con DAP $\geq$ $2.5 \mathrm{~cm}$, como lo hizo Gentry en sus estudios, se obtuvo un promedio de 30 ind/ 0.1 ha para Paujil y 27 ind/0.1 ha para Buenos Aires, por lo que la densidad en nuestros lugares de estudio es más bien menor. Estos resultados también ponen en evidencia la gran abundancia de individuos con diámetros entre $0.5 \mathrm{y}$ $2.5 \mathrm{~cm}$ (112 y $72 / 0.1$ ha).

Tabla 1. Fórmulas del Índice de Valor de Importancia de Lianas/Hemiepífitas (IVIL), Índice de Valor de Importancia de Familias de Lianas/Hemiepífitas (IVIFL), Índice de Diversidad de Shannon (H') e Índice de Diversidad $\alpha$ de Fisher (S).

\begin{tabular}{|c|c|}
\hline ÍNDICE & FÓRMULA \\
\hline $\begin{array}{l}\text { Índice de Valor de } \\
\text { Importancia de } \\
\text { Lianas/Hemiepífitas } \\
\text { (IVIL) } \\
\text { Valor de Importancia de } \\
\text { las Familias de } \\
\text { Lianas/Hemiepífitas } \\
\text { (IVIFL) }\end{array}$ & $\begin{array}{l}=\text { Densidad relativa }+ \text { Dominancia } \\
\text { relativa }+ \text { Frecuencia relativa }+ \\
\text { Longitud relativa (Romero-Saltos, } \\
1999) \\
=\text { Densidad relativa }+ \text { Dominancia } \\
\text { relativa }+ \text { Diversidad relativa }+ \\
\text { Longitud relativa (Romero-Saltos, } \\
1999) \\
\mathrm{S}=\alpha \ln (\mathrm{N} / \alpha) \\
\text { donde } \mathrm{S} \text { es el número de especies, } \mathrm{N} \\
\text { el número de individuos, y } \alpha \text { es el } \\
\text { índice de diversidad mismo (Berry, } \\
2002) \\
\mathrm{H}^{\prime}=-\Sigma \mathrm{P}_{\mathrm{i}} \text { ln } \mathrm{P}_{\mathrm{i}} \\
\text { donde } \mathrm{P}_{\mathrm{i}} \text { es la proporción con que } \\
\text { cada especie aporta al total de } \\
\text { individuos (Magurran, 1988) }\end{array}$ \\
\hline
\end{tabular}

Respecto a las especies que más contribuyen con las densidades mencionadas al principio, las hemiepífitas Philodendron sp.1 y Asplundia sp. 1 se encuentran en primer lugar en la E.B. Paujil, y Philodendron ernestii Engl. y Philodendron wittianum Engl. en la C.N. Buenos Aires (Tabla 2). Las especies que siguen en abundancia son lianas leñosas: Abuta sp.1, Abuta sp.2 y Croton sampatik Müll. Arg. en la E.B. Paujil, y Stizophyllum inaequilaterum Bureau \& K. Schum. en la C.N. Buenos Aires; éstas serían entonces las más abundantes si es que el límite mínimo de DAP registrado sería de $2.5 \mathrm{~cm}$, pues sus tallos suelen ser superiores a esta medida.

A nivel de familias, el $46 \%$ de los individuos muestreados en Paujil pertenecen únicamente a 4 familias: Araceae, Bignoniaceae, Menispermaceae y Fabaceae, (Tabla 3a). Del mismo modo, en Buenos Aires estas 4 familias son las más representativas, abarcando el $55 \%$ de los individuos registrados, aunque en orden diferente (Tabla $3 \mathrm{~b}$ ). Treinta y dos familias completan el $54 \%$ de individuos restantes en Paujil, y 37 representan el $45 \%$ de los individuos encontrados en Buenos Aires. Así, se puede ratificar la afirmación de Gentry (1991) para el Neotrópico en general: "a pesar de que el hábito escandente evolucionó en varios taxones por igual, la gran 
mayoría de trepadoras pertenecen a pocas familias". Es importante aclarar que este autor no incluyó dentro de las familias predominantes a las Aráceas, lo cual está relacionado con lo que se menciona más arriba respecto al mayor límite de DAP usado por él, ya que las especies hemiepífitas señaladas antes con mayor densidad y menor diámetro pertenecen a esta familia.

Tabla 2. Índice de Valor de Importancia de Lianas y Hemiepífitas de las 10 especies que registraron los mayores valores en (a) E.B. Paujil, y (b) C.N. Buenos Aires.

\begin{tabular}{|c|c|c|c|c|c|}
\hline \multicolumn{6}{|c|}{ 2(a) } \\
\hline ESPECIE & $\begin{array}{l}\text { DENS. } \\
\text { REL. } \\
(\%)\end{array}$ & $\begin{array}{l}\text { FREC. } \\
\text { REL. } \\
(\%)\end{array}$ & $\begin{array}{l}\text { DOM. } \\
\text { REL. } \\
(\%)\end{array}$ & $\begin{array}{l}\text { LONG. } \\
\text { REL. } \\
(\%)\end{array}$ & $I V I L$ \\
\hline Philodendron sp.1 & 17.470 & 5.271 & 4.779 & 10.122 & 37.642 \\
\hline $\begin{array}{l}\text { Croton sampatik } \\
\text { Müll. Arg. }\end{array}$ & 3.100 & 2.947 & 11.016 & 5.459 & 22.522 \\
\hline Abuta sp. 2 & 3.031 & 3.258 & 7.524 & 3.689 & 17.502 \\
\hline Abuta sp.1 & 2.935 & 2.267 & 4.937 & 3.836 & 13.975 \\
\hline Ficus sp. 1 & 0.242 & 0.340 & 8.578 & 0.551 & 9.710 \\
\hline Marcgravia sp.2 & 3.027 & 2.947 & 0.430 & 2.758 & 9.162 \\
\hline Asplundia sp.2 & 3.480 & 2.381 & 0.475 & 2.196 & 8.533 \\
\hline $\begin{array}{l}\text { Clusia hammeliana } \\
\text { Pipoly }\end{array}$ & 2.401 & 2.324 & 0.527 & 2.809 & 8.061 \\
\hline Distictella sp.1 & 2.170 & 1.984 & 1.202 & 2.696 & 8.053 \\
\hline $\begin{array}{l}\text { Machaerium } \\
\text { multifoliolatum Ducke }\end{array}$ & 0.959 & 1.332 & 3.716 & 1.317 & 7.324 \\
\hline \multicolumn{6}{|c|}{2 (b) } \\
\hline ESPECIE & $\begin{array}{c}\text { DENS. } \\
\text { REL. } \\
(\%)\end{array}$ & $\begin{array}{l}\text { FREC. } \\
\text { REL. } \\
(\%)\end{array}$ & $\begin{array}{l}D O M . \\
R E L . \\
(\%)\end{array}$ & $\begin{array}{l}\text { LONG. } \\
\text { REL. } \\
\text { (\%) }\end{array}$ & $I V I L$ \\
\hline $\begin{array}{l}\text { Philodendron ernestii } \\
\text { Engl. }\end{array}$ & 5.723 & 3.816 & 1.210 & 2.619 & 13.367 \\
\hline $\begin{array}{l}\text { Deguelia scandens } \\
\text { Aubl. }\end{array}$ & 1.650 & 1.714 & 7.434 & 2.268 & 13.066 \\
\hline $\begin{array}{l}\text { Stizophyllum } \\
\text { inaequilaterum } \\
\text { Bureau \& K. Schum. }\end{array}$ & 3.443 & 1.789 & 4.103 & 3.561 & 12.896 \\
\hline $\begin{array}{l}\text { Clusia hammeliana } \\
\text { Pipoly }\end{array}$ & 2.821 & 3.078 & 1.477 & 2.920 & 10.296 \\
\hline Maripa sp.1 & 0.733 & 0.402 & 6.799 & 1.764 & 9.697 \\
\hline $\begin{array}{l}\text { Gnetum nodiflorum } \\
\text { Brongn. }\end{array}$ & 0.271 & 0.327 & 8.101 & 0.659 & 9.358 \\
\hline $\begin{array}{l}\text { Acacia kuhlmannii } \\
\text { Ducke }\end{array}$ & 1.099 & 0.803 & 5.777 & 1.113 & 8.792 \\
\hline Xylophragma sp.1 & 1.992 & 1.382 & 2.807 & 2.393 & 8.573 \\
\hline $\begin{array}{l}\text { Philodendron } \\
\text { wittianum Engl. }\end{array}$ & 3 & 6 & 8 & 1.888 & 8.315 \\
\hline Deguelia sp.1 & 1.760 & 1.611 & 1.945 & 2.667 & 7.984 \\
\hline
\end{tabular}

El hecho de que las hemiepífitas sean las especies más abundantes y se encuentren dentro de las familias predominantes en ambas localidades puede estar relacionado con variables ambientales como la humedad (Gentry, 1991), la cual resulta su principal fuente de agua ya que en un comienzo no pueden obtenerla del suelo como el resto de plantas, pues inician su ciclo vital como epífitas (Putz \& Holbrook, 1986).
Frecuencia

La mayoría de las especies de ambas localidades parecen estar espacialmente restringidas o agregadas, pues registraron una elevada frecuencia en sólo una de las subunidades de los transectos que se evaluaron (Figura 2). Se ha comprobado que las lianas, debido a su reproducción vegetativa, presentan un patrón de crecimiento agregado (Putz, 1984b; Putz \& Chai, 1987; Gentry, 1985, 1991; Ibarra-Manríquez \& Martínez-Ramos, 2002), lo cual es ratificado por los resultados obtenidos. De acuerdo a esta afirmación, se podría esperar que las especies con bajas frecuencias tengan una gran densidad, sin embargo, en ambos lugares se obtuvo un patrón más bien inverso (Figura 3 ), teniendo la mayoría de estas menos de 5 ind/0.1ha. Esto se debe, como se mencionó en la metodología, a que los transectos utilizados evitan justamente la sobre valoración de la densidad de las lianas (Gentry, 1985, 1991).

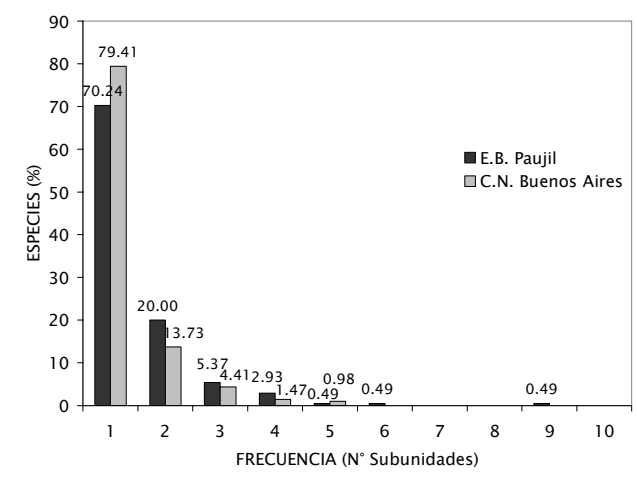

Figura 2. Distribución de la frecuencia de especies (en porcentaje) encontradas en los sitios de estudio, determinada como el número de subunidades $(2 \times 50$ $\mathrm{m})$ en las que se registraron. Las barras negras representan a la E.B. Paujil y las barras grises a la C.N. Buenos Aires.

Por otro lado, el hecho de que Philodendron sp. 1 registre una frecuencia elevada, anómala en comparación a la de las demás especies (Figura 3 a), puede deberse a que los grupos de individuos se establecen con mayor frecuencia en cualquier tipo de sustrato, sin preferir ninguno en particular, ya que su mecanismo de crecimiento por medio de raíces adventicias se lo permite; esto suele ocurrir con otro tipo de hemiepífitas (Putz \& Chai, 1987; Hegarty \& Caballé, 1991), y podría explicar también la elevada abundancia de las hemiepífitas en general. 


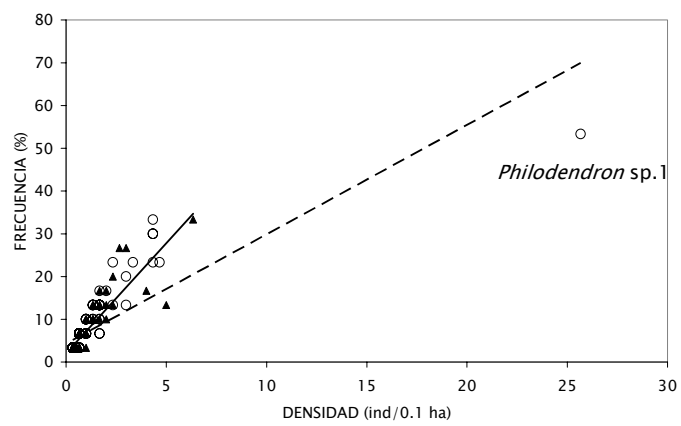

Figura 3. Distribución espacial de la abundancia de lianas y hemiepífitas en E.B. Paujil (círculos) y la C.N. Buenos Aires (triángulos). La línea de tendencia intermitente pertenece a la E.B. Paujil $(\mathrm{y}=2.5571 \mathrm{x}+$ $\left.4.3108, \mathrm{R}^{2}=0.6829, \mathrm{P}=6.63 \mathrm{E}-24\right)$, mientras que la continua pertenece a la C.N. Buenos Aires $(\mathrm{y}=$ $\left.5.1531 \mathrm{x}+2.052, \mathrm{R}^{2}=0.7883, \mathrm{P}=1.82 \mathrm{E}-16\right)$. Los valores de frecuencia están basados en el porcentaje de subunidades $(2 \times 50 \mathrm{~m})$ en los que la especie fue registrada. Se indica la posición de la hemiepífita Philodendron sp.1 perteneciente a la E.B. Paujil.
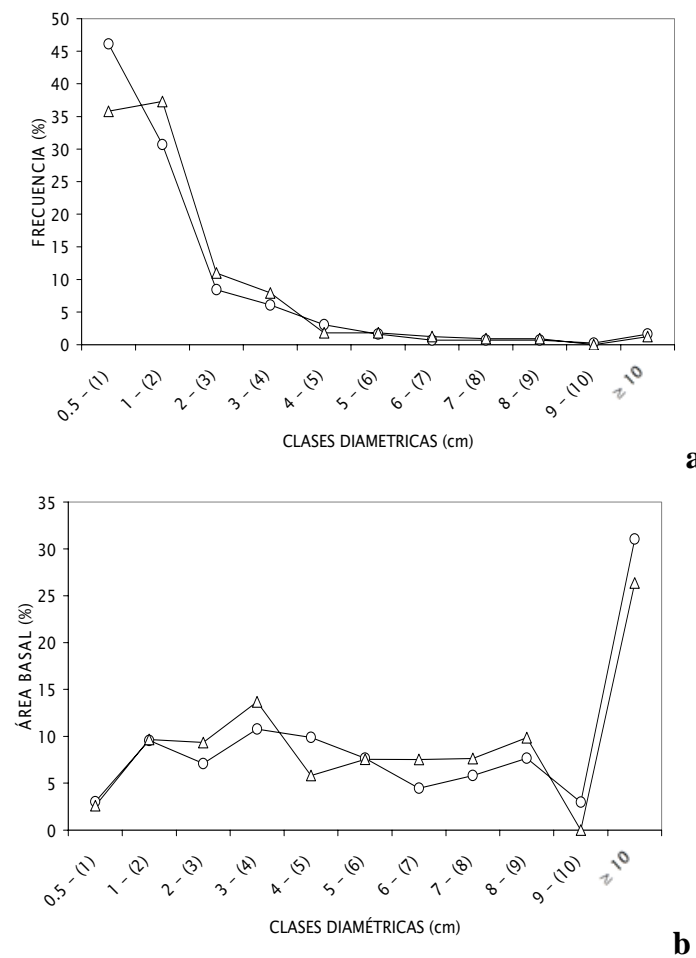

Figura 4. Distribución según clases diamétricas de (a) la frecuencia, y (b) el área basal de las lianas y hemiepífitas de la E.B. Paujil y la C.N. Buenos Aires, representadas por círculos $\mathrm{y}$ triángulos respectivamente.

\section{Dominancia}

El total de área basal por 0.1 ha para Paujil fue de $764.61 \mathrm{~cm}^{2}( \pm 215.12)$, mientras que en Buenos Aires fue de $590.97 \mathrm{~cm}^{2}( \pm 249.04)$.

La Figura 4a muestra la distribución de las especies por clase de tamaño para la E.B. Paujil y la C.N. Buenos Aires. En ambos casos la comunidad de lianas está conformada mayormente por lianas con diámetros menores a $2 \mathrm{~cm}$. Al analizar cuánto aportan estos individuos al área basal total de cada sitio (Figura $4 \mathrm{~b}$ ) se puede ver que representan menos del $15 \%$, mientras que los individuos con DAP $\geq 10 \mathrm{~cm}$ contribuyen con más del $25 \%$ al total del área basal de ambos lugares. Así, las especies más abundantes de cada sitio no son las más dominantes, ya que por ser hemiepífitas, el DAP es medido en las raíces aéreas que suelen ser menores a $1 \mathrm{~cm}$. Del mismo modo, especies de lianas y hemiepífitas leñosas como Gnetum nodiflorum Brongn. y Maripa sp. 2 en Buenos Aires, y Ficus sp.1 y Strychnos sp.1 en Paujil, que registraron diámetros cercanos a $10 \mathrm{~cm}$, se encuentran entre las especies más dominantes de sus respectivas localidades, habiendo sido representadas sólo por 1 o 2 individuos. Por lo tanto, es lógico afirmar que muchos individuos pequeños aporten menos al área basal total que pocos individuos con diámetros mayores (Romero-Saltos, 1999).

Tabla 3. Índice de Valor de Importancia de las 5 familias de plantas trepadoras con mayores valores en (a) E.B. Paujil, y (b) C.N. Buenos Aires.

\begin{tabular}{lccccc}
\hline \multicolumn{1}{c}{ FAMILIA } & DENS. & DOM. & DIV. & LONG. & IVIFL \\
& REL.(\%) & REL.(\%) & REL.(\%) & REL.(\%) & \\
\hline Araceae & 23.94 & 5.65 & 8.96 & 13.38 & 51.9 \\
Bignoniaceae & 8.62 & 7.32 & 10.94 & 11.96 & 38.8 \\
Menispermaceae & 7.19 & 12.81 & 3.88 & 7.79 & 31.7 \\
Fabaceae & 5.83 & 6.53 & 9.07 & 6.62 & 28.0 \\
Euphorbiaceae & 4.77 & 12.27 & 3.88 & 6.98 & 27.9 \\
\hline & & $3(\mathrm{~b})$ & & & \\
\hline \multicolumn{1}{c}{ FAMILIA } & DENS. & DOM. & DIV. & LONG. & IVIFL \\
\hline Bignoniaceae & 19.038 & 19.087 & 17.641 & 22.492 & 78.258 \\
Fabaceae & 12.343 & 24.086 & 12.272 & 15.085 & 63.785 \\
Araceae & 18.633 & 3.855 & 7.986 & 9.663 & 40.137 \\
Menispermaceae & 4.232 & 3.450 & 5.950 & 4.147 & 17.779 \\
Convolvulaceae & 2.350 & 8.178 & 2.631 & 3.790 & 16.950 \\
\hline
\end{tabular}

A nivel de familias, Menispermaceae y Fabaceae son las que presentan mayor área basal en Paujil y Buenos Aires, respectivamente (Tabla 3), ya que las dos familias poseen especies que se encuentran entre las más dominantes en sus respectivas localidades, como Abuta sp.1 y Abuta sp. 2 en Paujil, y Deguelia scandens Aubl. y Acacia kuhlmannii Ducke en Buenos Aires (Tabla 3). En general, las 10 familias con mayor dominancia representan el $82 \%$ del área 
basal de Paujil y el $81 \%$ de Buenos Aires, muy similares al resultado encontrado por Paz y Miño (1990, citado por Romero-Saltos, 1999) para un bosque bajo ecuatoriano: $83 \%$.

Longitud

No existen muchos estudios que hayan registrado la longitud de lianas o hemiepífitas, debido a la dificultad de medir esta cararacterísitica. Los trabajos que sí la han tomado en cuenta son aquellos que han descrito la estructura de la comunidad de trepadoras a través del Índice de Valor de Importancia de Lianas.

En la localidad de Paujil las especies que más contribuyeron con la longitud alcanzada fueron Philodendron sp.1, Croton sampatik Müll. Arg., Abuta sp. 1 y sp. 2 (Tabla 2 a). La primera especie, por ser una hemiepífita, no suele llegar al estrato más elevado del bosque pues necesita humedad para desarrollarse (Gentry, 1991), encontrándose ésta en mayor cantidad al interior de la cúpula del bosque; por lo tanto, su longitud promedio es de $6.5 \mathrm{~m}$. Su gran densidad hace que posea la mayor longitud acumulada. En cambio, para las otras 3 especies, por su condición de lianas leñosas, el recurso más importante es la luz (Putz, 1984 b); por eso, suelen crecer más rápido en longitud que en área basal (Putz, 1984 b; Phillips, 1991), llegando a tener extensiones elevadas con el propósito de alcanzar el dosel. Por lo tanto, a pesar de no haber sido tan abundantes, sus promedios de longitud son mayores $(10.2,8.9$ y $7.1 \mathrm{~m}$, respectivamente).

En el caso de Buenos Aires, sucede del mismo modo para las 4 especies con mayores longitudes, teniendo las lianas Stizophyllum inaequilaterum Bureau \& K. Schum. y Deguelia sp. 1, y la hemiepífita leñosa Clusia hammeliana Pipoly más longitud que la hemiepífita herbácea Philodendron ernestii Engl., siendo sus promedios $13 \pm 7.5 \mathrm{~m}, 19 \pm$ $8.6 \mathrm{~m}, 15 \pm 7.5 \mathrm{~m}$ y $6 \pm 3.2 \mathrm{~m}$, respectivamente. En este caso, si bien $P$. ernestii Engl.es la más abundante, no lo es lo suficiente como para superar a las lianas nombradas.

Consecuentemente, de acuerdo a las especies mencionadas, las familias que registraron mayor longitud en Paujil fueron Araceae, Menispermaceae y Euphorbiaceae, y Bignoniaceae, Fabaceae, Araceae y Clusiaceae en Buenos Aires (Tabla 3). Algunas de las familias más dominantes coincidieron en ser las que abarcan la mayor longitud (Bignoniaceae, Menispermaceae y Euphorbiaceae en Paujil, y Fabaceae y Bignoniaceae en Buenos Aires), es decir, las que presentan mayor tamaño, lo cual es lógico ya que son familias donde predominan las formas de vida leñosas.

Riqueza

Se comparó el promedio de especies que registró cada familia por transecto $(0.1 \mathrm{ha})$ de las localidades estudiadas con los resultados obtenidos por Gentry $(1985,1991)$ para lianas y hemiepífitas en los transectos que él instaló en 21 sitios de la Llanura Amazónica (Figura 5). Cuando se consideran todas las especies colectadas, Bignoniaceae y Fabaceae son las familias con más especies en ambos sitios, dominancia confirmada por Gentry $(1985,1991)$ y por otros estudios realizados hasta la fecha para muchos bosques bajos de la Amazonía, independientemente del tipo de suelo en el que se encuentren (Dewalt et al., 2000; Ibarra-Manríquez \& Martínez-Ramos, 2002; Nabe-Nielsen, 2002; Paz \& Miño, 1990 citado por Romero-Santos, 1999). La familia Araceae, no fue considerada por Gentry como predominante ya que solo se encontró en gran medida en una de las localidades evaluadas, justamente en la más húmeda. Esta variable ambiental podría explicar la alta presencia de especies de esta familia en los lugares evaluados.

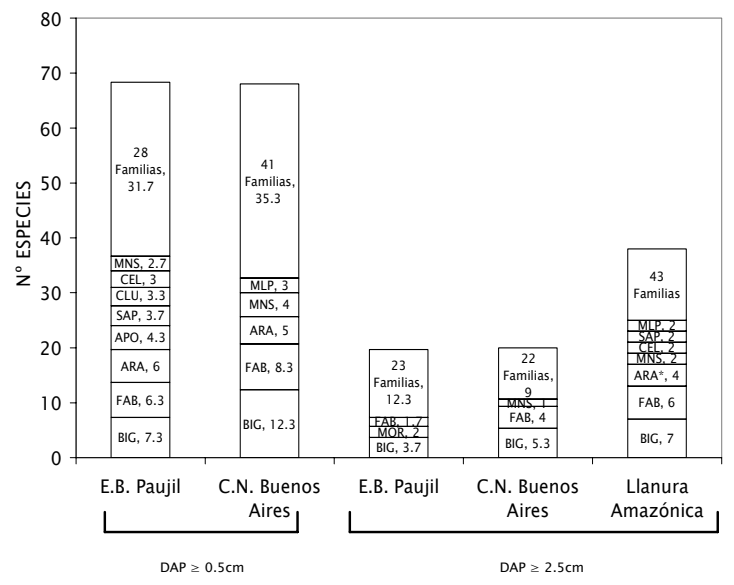

Figura 5. Promedio de riqueza de especies por familias en 0.1 ha en los sitios de estudio, primero incluyendo a todas las especies registradas (DAP $\geq 0.5$ $\mathrm{cm})$, luego incluyendo únicamente a las especies con DAP $\geq 2.5 \mathrm{~cm}$. Se comparan ambos resultados con el promedio obtenido por Gentry (1991) en 21 sitios de la Llanura Amazónica. $\mathrm{BIG}=$ Bignonaceae, $\mathrm{FAB}=$ Fabaceae, ARA $=$ Araceae, $\mathrm{APO}=$ Apocynaceae, $\mathrm{SAP}$ $=$ Sapindaceae, $\mathrm{CLU}=$ Clusiaceae, $\mathrm{CEL}=$ Celastraceae, $\mathrm{MNS}=$ Menispermaceae, $\mathrm{MLP}=$ Malpighiaceae, MOR $=$ Moraceae. $*$ Araceae sólo fue una familia importante en uno de los 21 sitios evaluados por Gentry (1991).

Por otro lado, cuando sólo se incluyen las especies con $\mathrm{DAP} \geq 2.5 \mathrm{~cm}$, a pesar de que el número de especies se reduce a menos de un tercio, como se menciona más arriba, la dominancia de Bignoniaceae continua en ambos sitios, mientras que Araceae deja de tener importancia. 
Índice de Valor de Importancia de Lianas y Hemiepífitas

La morfoespecie Philodendron sp. 1 fue la de mayor estructura en Paujil, teniendo los mayores valores para densidad, frecuencia y longitud (Tabla 2 a). $\mathrm{Su}$ dominancia no fue la más alta debido a que el DAP es medido en las raíces aéreas que presenta, las cuales suelen ser menores a $1 \mathrm{~cm}$. Como se comentó anteriormente, por ser ésta especie una Arácea, la humedad es un factor que puede haber sido determinante para su gran importancia en Paujil. Las siguientes especies con mayor importancia son las lianas Croton sampatik Müll. Arg., Abuta sp.1 y sp. 2, que tuvieron sobretodo valores sobresalientes de dominancia y longitud, siendo las lianas y en general trepadoras, de mayores dimensiones en este bosque.

En el caso de Buenos Aires, la Arácea Philodendron ernestii Engl. fue la que obtuvo un mayor valor de importancia (Tabla 2 b), teniendo, al igual que Philodendron sp.1, los valores más altos en cuanto a densidad y frecuencia. Las especies que siguieron en importancia fueron Deguelia scandens Aubl., Stizophyllum inaequilaterum Bureau \& K. Schum. y Clusia hammeliana Pipoly; las dos primeras son lianas que tuvieron los mayores valores en cuanto a dominancia y longitud, es decir que fueron las especies más grandes; en cambio la última, es una hemiepífita que predominó más a nivel de frecuencia, es decir, que tuvo una mayor distribución en los transectos.

Índice de Valor de Importancia de Familias

La familia que resultó ser más importante en Paujil fue Araceae (Tabla 3 a), debido probablemente a la abundancia elevada de Philodendron sp.1. Las familias siguientes en importancia fueron Bignoniaceae $y$ Menispermaceae, también con una alta densidad, pero sobre todo con gran dominancia y longitud, por lo que parece que las lianas que pertenecen a estas familias suelen ser las de mayores dimensiones en el bosque. Bignoniaceae, por su parte, resultó ser la familia más diversa en cuanto a número de especies (Figura 5, Tabla 3 a), seguida por Fabaceae, por lo que también son consideradas familias estructuralmente importantes.

Para la localidad de Buenos Aires, Bignoniaceae y Fabaceae fueron las familias que ocuparon los primeros lugares en la mayoría de las variables evaluadas (Tabla $3 \mathrm{~b}$ ), siendo no sólo las familias más abundantes y con mayor distribución espacial, sino también las que poseen las lianas más grandes. La familia que le sigue es
Araceae, principalmente por su elevado valor de densidad, debido posiblemente a Philodendron ernestii Engl.

En resumen, se puede decir, que estos valores representan los de las especies más importantes para la estructura de ambos bosques. Por otro lado, el hecho de que Bignoniaceae y Fabaceae se encuentren entre las familias más importantes para las localidades estudiadas, coincide con el predominio de ambas familias en los bosques bajos amazónicos estudiados anteriormente (Gentry, 1985, 1991, 1993; Paz \& Miño, 1990 citado por Romero-Saltos, 1999; Dewalt et al., 2000; Nabe-Nielsen, 2002).

Diversidad

Los valores calculados del índice Shannon para la E.B. Paujil y la C.N. Buenos Aires fueron $3.69 \pm 0.28$ y $3.98 \pm 0.20$, respectivamente. Magurran (1988) afirma que el rango en el que suele obtenerse este índice es $1-4$, por lo que podría decirse que ambos lugares poseen diversidades elevadas. Probablemente el menor valor de diversidad para Paujil puede deberse a la presencia de la hemiepífita Philodendron sp.1 que resultó ser dominante debido a su elevada densidad. Del mismo modo, la C.N. Buenos Aires puede tener una mayor diversidad debido a que no parece haber dominancia de alguna de las especies registradas, y más bien, existe una mayor equidad entre todas ellas.

Tabla 4. Diversidad $\alpha$ de Fisher en los sitios de estudio en comparación con las localidades de Cuyabeno en Ecuador (Paz y Miño, 1990 citado por Romero-Saltos, 1999), Isla Barro Colorado en Panamá (DeWalt et al., 2000) y en las cercanías de Manaos, Brasil (Laurance et al., 2001).

\begin{tabular}{|c|c|c|c|c|c|c|}
\hline CARACTERÍSTICAS & $\begin{array}{l}\text { E.B. } \\
\text { PAUJIL }\end{array}$ & $\begin{array}{c}\text { C.N. } \\
\text { BUENOS } \\
\text { AIRES }\end{array}$ & CUYABENO & $\begin{array}{r}B A \\
C O L C\end{array}$ & $R A D O$ & MANAOS \\
\hline Tamaño de UM (ha) & 0.1 & 0.1 & 1 & 0.04 & 0.04 & 2.88 \\
\hline $\mathrm{DAP}(\mathrm{cm})$ & $\geq 0.5$ & $\geq 0.5$ & $\geq 0.5$ & $\geq 0.5$ & $\geq 0.5$ & $\geq 2$ \\
\hline $\begin{array}{l}\text { Hemiepífitas } \\
\text { incluidas }\end{array}$ & Sí & Sí & Sí & No & No & No \\
\hline Especies (N) & 68 & 68 & 98 & 31 & 23 & 83 \\
\hline Individuos (S) & 142 & 109 & 2160 & 265 & 139 & 1023 \\
\hline $\begin{array}{l}\text { Precipitación (prom } \\
\text { anual en mm) }\end{array}$ & 4000 & 4000 & 3500 & \multicolumn{2}{|c|}{2600} & 2700 \\
\hline $\begin{array}{l}\text { Diversidad } \alpha \text { Fisher } \\
\text { (DAP } \geq 0.5 \mathrm{~cm}, \text { con } \\
\text { hemiepífitas) }\end{array}$ & 52 & 82.48 & & & & \\
\hline $\begin{array}{l}\text { Diversidad } \alpha \text { Fisher } \\
\text { (DAP } \geq 0.5 \mathrm{~cm}, \text { sin } \\
\text { hemiepífitas } \\
\text { herbáceas) }\end{array}$ & 49.45 & 78.50 & 21.14 & & & \\
\hline $\begin{array}{l}\text { Diversidad } \alpha \text { Fisher } \\
\text { (DAP } \geq 0.5 \mathrm{~cm} \text {, sin } \\
\text { hemiepífitas) }\end{array}$ & 49.31 & 93.53 & & 9.87 & 7.99 & \\
\hline $\begin{array}{l}\text { Diversidad } \alpha \text { Fisher } \\
\text { (DAP } \geq 2 \mathrm{~cm} \text {, sin } \\
\text { hemiepífitas) }\end{array}$ & 32.58 & 37.97 & & & & 21.33 \\
\hline
\end{tabular}

Para poder comparar la diversidad encontrada en este estudio con otros, se determinó el índice de 
diversidad $\alpha$ de Fisher para ambas localidades (Tabla 4). Dado que este índice no está influenciado por el tamaño de unidad muestral (Magurran, 1988), y por lo tanto, permite hacer comparaciones entre parcelas de diferente área y número de individuos (Berry, 2002), los valores obtenidos fueron contrastados con los calculados en diferentes investigaciones realizadas en bosques neotropicales. Por un lado, la Reserva Cuyabeno en Ecuador (Paz \& Miño, 1990 citados por Romero-Santos, 1999), en donde, al igual que en nuestro estudio, se incluyeron los individuos de por lo menos $0.5 \mathrm{~cm}$ y hemiepífitas leñosas, presentó un valor bastante bajo de diversidad (Tabla 4) en comparación con nuestros lugares de estudio. Al parecer, el haber excluido a las hemiepífitas herbáceas en Cuyabeno (a excepción de 2 especies) ha influido considerablemente en este resultado, pues como se ha dicho antes, este tipo de trepadoras fue abundante y de gran diversidad tanto en Paujil como en Buenos Aires. Para realizar una mejor comparación excluimos esas especies en los cálculos de diversidad de nuestras localidades, con lo cual se obtuvieron valores un poco más bajos, pero aún mucho mayores que el registrado para Cuyabeno (Tabla 4).

Por su parte, en el estudio realizado en el Monumento Nacional de Barro Colorado en Panamá (DeWalt et al., 2000) también se consideró el mismo diámetro, pero no fueron tomadas en cuenta las hemiepífitas. Eliminando en este caso este tipo de planta trepadora de nuestro análisis, se obtiene que los valores de diversidad siguen siendo superiores a la diversidad de Barro Colorado (Tabla 4). En el caso de Manaos, Brasil (Laurance et al., 2001) (Tabla 4) además de excluir las hemiepífitas sólo se tomaron en cuenta las plantas trepadoras con un diámetro igual o superior a $2 \mathrm{~cm}$, restricción que elimina a un gran número de lianas, ya que tanto en Paujil como en Buenos Aires existe una cantidad importante de trepadoras que se encuentran en la clase diamétrica 0.5 - $2.5 \mathrm{~cm}$. En este caso la diversidad de nuestras localidades también fue superior, incluso cuando se hizo el cálculo sin tomar en cuenta hemiepífitas, ni lianas con un DAP menor a $2 \mathrm{~cm}$ (Tabla 4).

En resumen, a pesar de que sometamos nuestros datos a las mismas restricciones utilizadas en los trabajos mencionados, las diversidades de nuestros lugares de estudio siempre resultaron ser superiores (Tabla 4). Se puede decir entonces que la metodología no es un factor que haya influido en las diferencias, por lo tanto es muy posible que sean variables ambientales propias de cada sitio las que estén interviniendo en estos resultados. Al respecto, Gentry (1988) y Clinebell et al. (1995) demostraron que existe una correlación positiva entre la diversidad de plantas y la precipitación, en localidades del Neotrópico. De esta manera, la elevada precipitación existente en Paujil y Buenos Aires a comparación del resto de lugares de estudio (Tabla 4) mencionados podría estar determinando su mayor diversidad.

Por otro lado, los trabajos con los que se comparó este estudio no especifican el tipo de suelo ni la humedad de dichas localidades, por lo que no se puede suponer si existe alguna relación de estas variables con la diversidad encontrada. Sin embargo, como Clinebell et al. (1995) afirmaron que es posible que la fertilidad del suelo no tenga un efecto directo en la riqueza de especies de varios localidades neotropicales, se podría decir que la mayor diversidad encontrada en nuestros lugares de estudio se debe principalmente a la precipitación.

\section{Conclusiones}

En relación a la estructura de las comunidades de trepadoras, la predominancia de hemiepífitas herbáceas Philodendron sp.1 y Philodendron ernestii Engl. puede deberse a adaptaciones altamente efectivas en su medio, como lo son sus raíces adventicias que les facilitan comenzar su desarrollo en cualquier tipo de sustrato, y también a variables ambientales, como la humedad relativa o la precipitación.

Las familias Bignoniaceae y Fabaceae fueron las más importantes a nivel estructural en ambas localidades debido a la mayor riqueza de especies que las conforman, lo que concuerda con lo establecido para bosques tropicales de bajío. Es posible que condiciones de mayor humedad en la E.B. Paujil hayan determinado la predominancia de la familia Araceae, debido a la gran abundancia de sus especies.

Finalmente, es posible que los altos valores de diversidad encontrados tanto en la E.B. Paujil como en la C.N. Buenos Aires, estén relacionados con variables ambientales, particularmente con la elevada precipitación registrada en estos lugares. De esta manera, se podría afirmar que los factores ambientales juegan un papel importante en la estructura y diversidad de comunidades de lianas, por lo que sería necesario realizar futuras investigaciones que tomen en cuenta estas relaciones.

\section{Agradecimientos}

Agradecemos al Fondo Christensen y al Jardín Botánico de Missouri - Perú por proporcionar la beca con la cual se pudo realizar esta investigación. Rodolfo Vásquez, Rocío Rojas y Abel Monteagudo aportaron la ayuda necesaria en la logística y determinación de los especimenes colectados. Apreciamos la colaboración de Juan Mateo, Agustín Quicha, Aurora Muellen, Adilio Fernández y las autoridades de la Comunidad Nativa Buenos Aires que nos permitieron realizar esta investigación en su comunidad. Saara DeWalt nos facilitó información no publicada para la evaluación de lianas. José Luis Mateo, Abad Utani y Tomas Ciriaco nos proporcionaron valiosa ayuda en campo. Edgardo 
Ortiz, Jorge Lingán y Franco Mellado colaboraron en la determinación de las especies de plantas trepadoras de las familias Ericaceae, Araceae y división Pteridophyta, respectivamente. Finalmente agradecemos a María de los Ángeles La TorreCuadros quien nos proporcionó valiosas sugerencias durante todas las etapas de esta investigación, y a John Bunce por la revisión del manuscrito.

\section{Literatura citada}

Berry P. 2002. Diversidad y endemismo en los bosques tropicales de bajura. En: Guariguata, M.R. \& Kattan, H, (Eds.). Ecología y conservación de bosques tropicales. Editorial Libro Universitario Regional, Costa Rica.

Bongers F., Schnitzer S.A. \& Traore D. 2002. The importance of lianas and consequences for forest management in West Africa. Bioterre, Rev. Inter. Sci. de la Vie et de la Terre, $\mathrm{N}^{\mathrm{o}}$ special.

Brack A. 1984. Plan Maestro del Parque Nacional Yanachaga Chemillén. INADE, USAID / PEPP, PDR Palcazu. Iscazacin - Oxapampa, Perú.

Burnham R.J. 2002. Dominance, diversity and distribution of lianas in Yasuní, Ecuador: who is on top? Journal of Tropical Ecology . 18: 845-864.

Clark D.B. \& Clark D.A. 1990. Distribution and Effects on Tree Growth of Lianas and Woody Hemiepiphytes in a Costa Rican Tropical Wet Forest. Journal of Tropical Ecology. 6: 321-331.

Clinebell R.R., Phillips O.L., Gentry A.H., Stark N. \& Zuuring H. 1995. Prediction of neotropical tree and liana species richness from soil and climatic data. Biodiversity and Consevation. 4: 56-90.

DeWalt S.J..Schnitzer S.A \& Denslow J.S. 2000. Density and diversity of lianas along a chronosequence in a central Panamanian lowland forest. Journal of Tropical Ecology. 16:1-19.

Emmons L.H. \& Gentry A.H. 1983. Tropical forest structure and the distribution of gliding and prehensile-tailed vertebrates. American Naturalist. 121: 513-524.

Gentry A.H. 1983. Lianas and the "paradox" of contrasting latitudinal gradients in wood and litter production. Tropical Ecology. 24: 63-67.

. 1985. An ecotaxonomic survey of Panamanian lianas. En: W. D'Arcy \& M. Correa, eds. The botany and natural history of Panama: La botánica e historia natural de Panamá. Pp. 29-42. Monographs in Systematic Botany 10, Missouri Botanical Garden, USA.

1991. The distribution and evolution of climbing plants. En: En: F.E. Putz \& H.A. Mooney, eds.The Biology of Vines. Pp. 3-52. Cambridge University Press, Cambridge, England.

1993. A field guide to the families and genera of Woody Plants of Northwest South América (Colombia, Ecuador, Perú). The University of Chicago Press. Chicago and London.
Gerwing J.J., Schnitzer S.A., Burnham R.J., Bongers F., Chave J., DeWalt S.J., Ewango C.E.N., Foster, Kenfack D., Martínez-Ramos M., Parren M., Parthasarathy N., Pérez-Salicrup D.R., Putz F.E. \& Thomas D.W. 2006. A Standard Protocol for Liana Censuses. Biotropica. 38 (2): 256-261.

Hegarty E.E. \& Caballé G. 1991. Distribution and abundance of vines in forest communities. En: En: F.E. Putz \& H.A. Mooney, eds.The Biology of Vines. Pp. 313-335. Cambridge University Press, Cambridge, England.

Honorio E., Phillips O. \& Cazorla M.Y. 2003. Manual de Campo para la evaluación de la infección de Lianas en un Parcela Permanente. Rainfor.

Ibarra-Manríquez G. \& Martínez-Ramos M. 2002. Landscape variation of liana communities in a Neotropical rain forest. Plant Ecology. 160: 91-112.

Instituto del Bien Común. 2006. Atlas de Comunidades Nativas de la Selva Central. Lima,. Delamónica P.M. Fearnside, S. D'Angelo, A. Jerozolinski, L. Pohl \& T.E. Lovejoy. 2001. Rain forest fragmentation and the structure of amazonianan liana communities. Ecology, 82 (1). pp: $105-116$.

Magurran, A.E. 1988. Diversidad Ecológica y su Medición. Princeton University Press, Princeton, New Jersey.

Nabe-Nielsen J. 2000. Liana Community and Population Ecology in a Neotropical Rain Forest. Ph.D. dissertation submitted to the faculty of Natural Sciences, Aarhus University, Denmark.

ONERN. 1995. Mapa Ecológico del Perú. Ministerio de Agricultura. Lima, Perú.

Pérez-Salicrup D. \& Sork V. 2001. Lianas and Trees in a Liana Forest of Amazonian Bolivia. Biotropica. 33(1): 34-47.

Phillips O.L. \& Miller J.S., eds. 2002. Global patterns of plant diversity: Alwyn H. Gentry's forest transect data set. Missouri Botanical Garden Press.

Putz F.E. 1983. Liana Biomass and Leaf Area of a "Terra Firme" Forest in the Rio Negro Basin, Venezuela. Biotropica. 15(3): 185-189.

-.1984 a. How Trees Avoid and Shed Lianas. Biotropica .16(1): 19-23.

- 1984 b. The Natural History of Lianas on Barro Colorado Island, Panama. Ecology 65(6): 1713-1724.

Putz F.E. \& P. Chai. 1987. Ecological Studies of Lianas in Lambir National Park, Sarawak, Malaysia. Journal of Ecology. 75: 523-531.

Putz F.E. \& Holbrook N. 1986. Notes on the Natural History of Hemiepiphytes. Selbyana.9: 61-69.

Romero-Saltos H.G. 1999. Diversidad, análisis estructural y aspectos florísticos relevantes de las lianas en una parcela de bosque muy húmedo premontano, Amazonía Ecuatoriana. Tesis de Licenciatura en Ciencias Biológicas, Pontificia Universidad Católica del Ecuador. Quito, Ecuador. 
Diciembre 2006

\section{Apéndices}

Apéndice I. Lista de especies y morfoespecies de plantas trepadoras determinadas para la E.B. Paujil y la C.N. Buenos Aires, con la forma de vida y el número total de individuos de cada especie por localidad. $* 1=1$ liana, e $=$ enredadera, $\mathrm{h}=$ hemiepífita.

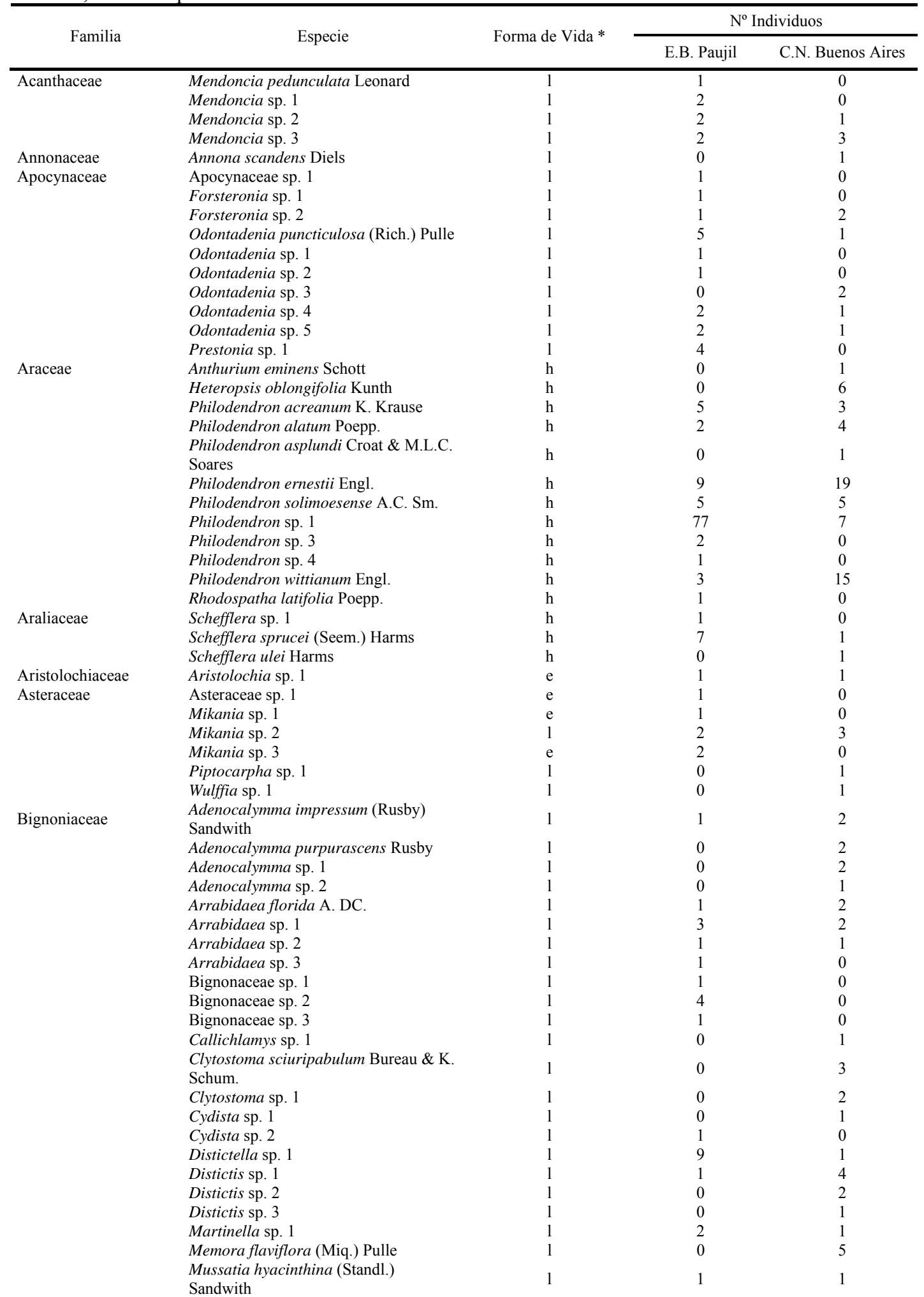


Blechnaceae Boraginaceae

Campanulaceae Celastraceae

Clusiaceae

Commelinaceae Connaraceae

Convolvulaceae

Cucurbitaceae

Dryopteridaceae

Cyclanthaceae
Combretaceae

Paragonia pyramidata (Rich.) Bureau Pithecoctenium sp. 1

Pleonotoma melioides (S. Moore) A.H. Gentry

Spathicalyx duckei (A. Samp.) A.H. Gentry

Stizophyllum inaequilaterum Bureau \& K. Schum.

Tabebuia serratifolia (Vahl) G.

Nicholson

Tanaecium nocturnum (Barb. Rodr.)

Bureau \& K. Schum.

Tynanthus panurensis (Bureau)

Sandwith

Tynanthus sp. 1

Xylophragma sp. 1

Xylophragma sp. 2

Salpichlaena volubilis (Kaulf.) J. Sm.

Boraginaceae sp. 1

Tournefortia sp. 1

Centropogon granulosus C. Pres

Celastraceae sp. 1

Celastraceae sp. 2

Cheiloclinium sp. 1

Cheiloclinium sp. 3

Hippocratea sp. 1

Hippocratea volubilis $\mathrm{L}$.

Salacia impressifolia (Miers) A.C. Sm.

Salacia sp. 2

Salacia sp. 4

Salacia sp. 5

Tontelea sp. 2

Tontelea sp. 3

Clusia hammeliana Pipoly

Clusia sp. 1

Clusia sp. 2

Clusia sp. 3

Clusia sp. 4

Clusiella axillaris (Engl.) Cuatrec.

Havetiopsis flexilis Spruce ex Planch. \&

Triana

Havetiopsis sp.

Combretum laxum Jacq.

Combretum sp. 1

Combretum sp. 2

Combretum sp. 3

Dichorisandra ulei J.F. Macbr.

Connarus sp. 1

Connarus sp. 2

Pseudoconnarus sp. 1

Rourea sp. 1

Convolvulaceae sp. 1

Convolvulaceae sp. 2

Dicranostyles holostyla Ducke Ipomoea philomega (Vell.) House Maripa axilliflora Mart. ex Meisn. Maripa sp. 1

Cayaponia ophthalmica R.E. Schult. Gurania rhizantha (Poepp. \& Endl.) C. Jeffrey

Gurania sp. 1

Siolmatra sp. 1

Asplundia sp. 1

Asplundia sp. 2

Doliocarpus sp. 1 Lam.

Doliocarpus sp. 2

Doliocarpus sp. 3

Doliocarpus sp. 4

Doliocarpus sp. 5

Pinzona sp. 1

Tetracera sp. 1

Lomariopsis nigropaleata Holttum Polybotrya caudata Kunze

\begin{tabular}{|c|c|}
\hline 1 & 0 \\
\hline 1 & 0 \\
\hline 1 & 0 \\
\hline 1 & 5 \\
\hline 1 & 1 \\
\hline 1 & 0 \\
\hline 1 & 0 \\
\hline 1 & 1 \\
\hline 1 & 0 \\
\hline 1 & 1 \\
\hline 1 & 1 \\
\hline h & 1 \\
\hline 1 & 0 \\
\hline 1 & 0 \\
\hline 1 & 0 \\
\hline 1 & 1 \\
\hline 1 & 1 \\
\hline 1 & 0 \\
\hline 1 & 2 \\
\hline 1 & 0 \\
\hline 1 & 1 \\
\hline 1 & 0 \\
\hline 1 & 1 \\
\hline 1 & 1 \\
\hline 1 & 0 \\
\hline 1 & 1 \\
\hline 1 & 1 \\
\hline $\mathrm{h}$ & 10 \\
\hline h & 1 \\
\hline $\mathrm{h}$ & 2 \\
\hline $\mathrm{h}$ & 1 \\
\hline $\mathrm{h}$ & 0 \\
\hline h & 1 \\
\hline h & 1 \\
\hline h & 1 \\
\hline 1 & 3 \\
\hline 1 & 0 \\
\hline 1 & 2 \\
\hline 1 & 2 \\
\hline e & 1 \\
\hline 1 & 2 \\
\hline 1 & 0 \\
\hline 1 & 0 \\
\hline 1 & 1 \\
\hline 1 & 0 \\
\hline 1 & 2 \\
\hline 1 & 0 \\
\hline 1 & 0 \\
\hline 1 & 1 \\
\hline 1 & 0 \\
\hline 1 & 1 \\
\hline 1 & 1 \\
\hline 1 & 1 \\
\hline 1 & 0 \\
\hline $\mathrm{h}$ & 14 \\
\hline $\mathrm{h}$ & 3 \\
\hline 1 & 0 \\
\hline 1 & 0 \\
\hline 1 & 2 \\
\hline 1 & 1 \\
\hline 1 & 0 \\
\hline 1 & 0 \\
\hline 1 & 2 \\
\hline $\mathrm{h}$ & 5 \\
\hline $\mathrm{h}$ & 2 \\
\hline
\end{tabular}




\begin{tabular}{|c|c|}
\hline & Polybotrya crassirhizoma Lellinger \\
\hline & Polybotrya osmundacea Humb. \& \\
\hline & Bonpl. ex Willd. \\
\hline & Polybotrya pubens Mart. \\
\hline Ericaceae & Cavendishia tarapotana var. \\
\hline Encacede & tarapotana (Meisn.) Benth. \& Hook.f. \\
\hline & Psammisia coarctata (Ruiz \& Pav. ex \\
\hline & D. Don) A. C. Sm. \\
\hline & Satyria panurensis (Benth. es Meisn.) \\
\hline & Benth. \& Hook.f. ex Nied. \\
\hline Euphorbiaceae & Croton sampatik Müll. Arg. \\
\hline & Mabea pulcherrima Müll. Arg. \\
\hline & Mabea sp. 2 \\
\hline & Manihot brachyloba Müll. Arg. \\
\hline & Plukenetia polyadenia Müll. Arg. \\
\hline Fabaceae & Acacia kuhlmannii Ducke \\
\hline & Acacia loretensis J.F. Macbr. \\
\hline & Bauhinia guianensis Aubl. \\
\hline & Bauhinia sp. 1 \\
\hline & Bauhinia sp. 3 \\
\hline & Dalbergia sp. 3 \\
\hline & Dalbergia sp. 5 \\
\hline & Deguelia scandens Aubl. \\
\hline & Deguelia sp. 1 \\
\hline & Deguelia sp. 2 \\
\hline & Deguelia sp. 3 \\
\hline & Deguelia sp. 4 \\
\hline & Deguelia sp. 5 \\
\hline & Derris utilis (A.C. Sm.) Ducke \\
\hline & Dioclea sp. 1 \\
\hline & Machaerium cuspidatum Kuhlm. \& \\
\hline & Hoehne \\
\hline & Machaerium floribundum Benth. \\
\hline & Machaerium multifoliolatum Ducke \\
\hline & Machaerium sp. 1 \\
\hline & Machaerium sp. 2 \\
\hline & Machaerium sp. 3 \\
\hline & Machaerium sp. 4 \\
\hline & Machaerium sp. 5 \\
\hline & Macrolobium sp. 2 \\
\hline & Mimosa guilandinae (DC.) Barneby \\
\hline & Mimosa myriadenia (Benth.) Benth. \\
\hline & Mimosa rufescens Benth. \\
\hline & Piptadenia sp. 1 \\
\hline & Piptadenia sp. 2 \\
\hline Gnetaceae & Gnetum nodiflorum Brongn. \\
\hline Icacinaceae & Leretia cordata Vell. \\
\hline & Pleurisanthes sp. 1 \\
\hline & Pleurisanthes sp. 2 \\
\hline Lamiaceae & Aegiphila sp. 2 \\
\hline & Aegiphila sp. 3 \\
\hline & Lamiaceae sp. 1 \\
\hline Loganiaceae & Strychnos guianensis (Aubl.) Mart. \\
\hline & Strychnos peckii B.L. Rob. \\
\hline & Strychnos sp. 1 \\
\hline Malpighiaceae & Banisteriopsis sp. 1 \\
\hline & Mascagnia sp. 1 \\
\hline & Mascagnia sp. 2 \\
\hline & Mezia sp. 1 \\
\hline & Mezia sp. 2 \\
\hline & Malpighiaceae sp. 2 \\
\hline & Stigmaphyllon sp. 1 \\
\hline & Stigmaphyllon sp. 2 \\
\hline Malvaceae & Byttneria sp. 1 \\
\hline Marantaceae & Ischnosiphon obliquus (Rudge) Körn. \\
\hline Marcgraviaceae & Marcgravia sp. 1 \\
\hline & Marcgravia sp. 2 \\
\hline & Marcgravia sp. 3 \\
\hline & Marcgravia sp. 5 \\
\hline & Marcgravia strenua J.F. Macbr. \\
\hline Melastomataceae & Adelobotrys macrantha Gleason \\
\hline & Blakea sp. 1 \\
\hline & Ossaea sp. 1 \\
\hline
\end{tabular}

h

h

$\mathrm{h}$

h

h

h

1

1

1

1

1

1

1

1

1

1

1

1

1

1

1

1
1 


\begin{tabular}{|c|c|c|c|c|}
\hline & Topobea sp. 1 & 1 & 1 & 0 \\
\hline & Topobea sp. 3 & 1 & 0 & 1 \\
\hline \multirow{12}{*}{ Menispermaceae } & Abuta rufescens Aubl. & 1 & 1 & 1 \\
\hline & Abuta sp. 1 & 1 & 13 & 3 \\
\hline & Abuta sp. 2 & 1 & 13 & 0 \\
\hline & Anomospermum sp. 1 & 1 & 0 & 1 \\
\hline & Cissampelos sp. 1 & e & 0 & 1 \\
\hline & Cissampelos tropaeolifolia DC. & $\mathrm{e}$ & 3 & 0 \\
\hline & $\begin{array}{l}\text { Curarea toxicofera (Wedd.) Barneby \& } \\
\text { Krukoff }\end{array}$ & 1 & 0 & 1 \\
\hline & Disciphania sp. 1 & $\mathrm{e}$ & 1 & 1 \\
\hline & Orthomene sp. 1 & 1 & 0 & 2 \\
\hline & Sciadotenia sp. 1 & 1 & 0 & 2 \\
\hline & $\begin{array}{l}\text { Sciadotenia toxifera Krukoff \& A.C. } \\
\text { Sm. }\end{array}$ & 1 & 0 & 1 \\
\hline & Telitoxicum sp. 1 & 1 & 0 & 2 \\
\hline \multirow[t]{8}{*}{ Moraceae } & Ficus americana Aubl. & $\mathrm{h}$ & 2 & 0 \\
\hline & Ficus paraensis (Miq.) Miq. & $\mathrm{h}$ & 2 & 0 \\
\hline & Ficus pertusa L. f. & $\mathrm{h}$ & 0 & 1 \\
\hline & Ficus sp. 1 & $\mathrm{~h}$ & 1 & 0 \\
\hline & Ficus sp. 2 & $\mathrm{~h}$ & 1 & 0 \\
\hline & Ficus sp. 4 & $\mathrm{~h}$ & 1 & 0 \\
\hline & Ficus sp. 5 & $\mathrm{~h}$ & 1 & 0 \\
\hline & Ficus trigonata $\mathrm{L}$. & $\mathrm{h}$ & 0 & 1 \\
\hline \multirow[t]{2}{*}{ Passifloraceae } & Dilkea sp. 1 & 1 & 0 & 1 \\
\hline & Passiflora sp. 2 & 1 & 0 & 1 \\
\hline Phytolaccaceae & Seguieria americana L. & 1 & 4 & 0 \\
\hline \multirow[t]{3}{*}{ Piperaceae } & Piper sp. 2 & 1 & 0 & 1 \\
\hline & Piper sp. 3 & 1 & 0 & 1 \\
\hline & Piper xanthostachyum C. DC. & $\mathrm{e}$ & 0 & 1 \\
\hline Polygalaceae & $\begin{array}{l}\text { Moutabea aculeata (Ruiz \& Pav.) } \\
\text { Poepp. \& Endl. }\end{array}$ & 1 & 0 & 1 \\
\hline \multirow[t]{5}{*}{ Polygonaceae } & Coccoloba mollis Casar. & 1 & 2 & 0 \\
\hline & Coccoloba sp. 1 & 1 & 1 & 1 \\
\hline & Coccoloba sp. 2 & 1 & 1 & 0 \\
\hline & Coccoloba sp. 3 & 1 & 1 & 0 \\
\hline & Coccoloba sp. 4 & 1 & 0 & 1 \\
\hline Rubiaceae & $\begin{array}{l}\text { Sabicea villosa Willd. ex Roem. \& } \\
\text { Schult. }\end{array}$ & 1 & 1 & 0 \\
\hline \multirow[t]{9}{*}{ Sapindaceae } & Paullinia alata G. Don & 1 & 4 & 0 \\
\hline & Paullinia cuneata Radlk. & 1 & 1 & 0 \\
\hline & Paullinia rhizantha Poepp. \& Endl. & 1 & 5 & 1 \\
\hline & Paullinia sp. 1 & 1 & 2 & 0 \\
\hline & Paullinia sp. 2 & 1 & 2 & 0 \\
\hline & Paullinia sp. 3 & 1 & 2 & 0 \\
\hline & Paullinia sp. 4 & 1 & 0 & 1 \\
\hline & Paullinia sp. 5 & 1 & 0 & 1 \\
\hline & Serjania sp. 1 & 1 & 2 & 0 \\
\hline Smilacaceae & Smilax febrifuga Kunth & $\mathrm{e}$ & 1 & 1 \\
\hline \multirow[t]{2}{*}{ Solanaceae } & Juanulloa sp. 1 & $\mathrm{~h}$ & 0 & 1 \\
\hline & $\begin{array}{l}\text { Lycianthes amatitlanensis (J.M. Coult. } \\
\& \text { Donn. Sm.) Bitter }\end{array}$ & $\mathrm{e}$ & 0 & 4 \\
\hline \multirow[t]{3}{*}{ Urticaceae } & Coussapoa sp. 1 & $\mathrm{~h}$ & 0 & 1 \\
\hline & Coussapoa sp. 2 & $\mathrm{~h}$ & 2 & 0 \\
\hline & $\begin{array}{l}\text { Urera baccifera (L.) Gaudich. ex } \\
\text { Wedd. }\end{array}$ & e & 0 & 1 \\
\hline \multirow{4}{*}{ Vitaceae } & Cissus flavifolia Lombardi & 1 & 0 & 3 \\
\hline & Cissus sp. 1 & 1 & 0 & 1 \\
\hline & Cissus sp. 2 & 1 & 0 & 1 \\
\hline & Cissus sp. 3 & 1 & 0 & 1 \\
\hline
\end{tabular}

${ }^{1}$ Bióloga Universidad Nacional Agraria La Molina. caissarevillaminaya@yahoo.com

${ }^{2}$ Biólogo, M.Sc.ProfesorPrincipal de FisiologíaVegetal.UNALM. acalderon@lamolina.edu.pe 